\title{
A ANÁLISE DE POLÍTICAS PÚBLICAS NA PERSPECTIVA DO MODELO DE COALIZÕES DE DEFESA
}

\section{Victor Manuel Barbosa Vicente}

Universidade Federal de Uberlândia (UFU)/Universidade de Brasília (UnB)

\section{ANÁLISE DE POLÍTICAS PÚBLICAS NA PERSPECTIVA DO MODELO DE COALIZÕES DE DEFESA}

Resumo: O propósito deste ensaio é ressaltar as contribuições que o Modelo de Coalizões de Defesa (MCD) pode proporcionar para a melhoria de nossa compreensão sobre os padrões de mudança em um contexto no qual as políticas públicas são cada vez mais multifuncionais, intersetoriais e transversais. Proposto por Paul A. Sabatier, o MCD parte de um conjunto de premissas e, por meio de um modelo teórico, visa explicar a formulação e as mudanças nas políticas públicas, tendo como base dois pressupostos fundamentais. O primeiro, enxerga as causas das mudanças nas políticas públicas como resultado das disputas entre as coalizões de defesa e o segundo entende a mudança como produto do papel exercido pelo aprendizado político no processo. Essa abordagem foi considerada para efeitos deste ensaio pela sua coerência interna e aplicabilidade no estudo de políticas públicas.

Palavras-chave: Políticas públicas, modelos de análise de políticas públicas, modelo de coalizões de defesa.

\section{ANALYSIS OF PUBLIC POLICIES IN A PERSPECTIVE OF ADVOCACY COALITION FRAMEWORK}

Abstract: The purpose of this paper is to highlight the contributions that the Advocacy Coalition Framework (ACF) may provide to improve our understanding of the patterns of change in a world in which public policies are increasingly multifunctional, cross-sectoral and cross-cutting. Proposed by Paul A. Sabatier ACF part of a set of assumptions and by means of a theoretical model, aims to explain the formulation and changes in public policies based on two fundamental assumptions. The first sees the causes of changes in public policies as a result of disputes between advocacy coalitions and the second is that change is the product of the role played by policy-oriented learning in the process. This approach was considered for the purposes of this essay for its internal coherence and applicability in the study of public policy.

Key words: Public policies, models of public policies analysis, advocacy coalition framework. 


\section{INTRODUÇÃO}

\subsection{Principais pressupostos}

Proposto por Paul A. Sabatier para estudar a formulação e os processos pelas quais as políticas públicas mudam ao longo do tempo, a abordagem do Advocacy Coalition Framework (ACF) ou Modelo de Coalizões de Defesa (MCD) vem sendo sistematicamente aprimorada desde que foi inicialmente desenvolvida em 1988 (SABATIER, 1988; 1998; 1999; 2007; SABATIER; JENKINSSMITH, 1993; WEIBLE; SABATIER; MCQUEEN, 2009).

Sabatier (1988, p. 139) define uma coalizão de defesa como

[...] pessoas de uma variedade de posições (representantes eleitos e funcionários públicos, líderes de grupos de interesse, pesquisadores, intelectuais e etc.), que (i) compartilham determinado sistema de crenças: valores, ideias, objetivos políticos, formas de perceber os problemas políticos, pressupostos causais e (ii) demonstram um grau não trivial das ações coordenadas ao longo do tempo.

Portanto, a cola que mantém uma coalizão de defesa unida é o compartilhamento de crenças dos seus membros sobre assuntos políticos fundamentais (WEIBLE; SABATIER; MCQUEEN, 2009).

$O$ MCD assume que os membros das coalizões vão discordar muitas vezes em questões menores, mas que essa divergência será limitada, e rejeita a possibilidade de coalizões de conveniência, motivadas por autointeresses de curto prazo, dominarem o cenário de produção das políticas públicas ao longo do tempo. Segundo Sabatier (2007), esses pressupostos têm um impacto duradouro sobre as orientações políticas dos membros das coalizões.

Uma vez formadas, as coalizões de defesa competem para traduzir suas crenças compartilhadas em políticas públicas ou programas governamentais, mobilizando recursos políticos e participando de processos contínuos de aprendizagem política. Esse processo contínuo de aprendizagem é denominado de policy-oriented learning ou aprendizado orientado à política pública (fator essencialmente cognitivo) (SABATIER; JENKINS-SMITH, 1993).

O MCD concebe 0 aprendizado orientado à política pública como espinha dorsal da dinâmica interna de um subsistema, sendo ele diretamente influenciado pela produção de pesquisa aplicada e pelo papel do debate técnico sobre aspectos críticos de políticas públicas. Portanto, o efeito cumulativo de estudos e conhecimentos tem grande influência no redirecionamento das políticas públicas (WEISS, 1977; 1983; SABATIER, 1978; HAAS, 1991; SABATIER; WEIBLE, 2007; WEIBLE, 2008).

Desta forma, o policy-oriented learning é entendido como a permanente alteração de pensamentos e de comportamentos resultantes da experiência prática apoiada pela evolução do conhecimento técnico/cientifico do problema, o que permite revisões nos objetivos da política pública (SABATIER, 1998).

0 aprendizado permite que novos argumentos ou recursos sejam adicionados às discussões. Deste modo, segundo Sabatier (1978) e Engel (2007), à medida que esses novos argumentos técnicos surgem a partir de pesquisas desenvolvidas, o sistema de crenças dos atores de uma determinada coalizão tende a se fortalecer.

Nesse sentido, o espaço ou o ambiente para essas interações e para a ocorrência do processo político de aprendizagem é chamado de subsistema de políticas públicas (HOWLETT; RAMESH, 1998).

Sabatier descreve um subsistema de políticas públicas como um conjunto de atores individuais ou coletivos de uma variedade de organizações públicas, privadas e da sociedade civil (ONGs), num determinado escopo geográfico, que está ativamente preocupado com determinada questão de política pública e que sistematicamente tenta influenciar as decisões naquele domínio (SABATIER; JENKINS-SMITH, 1988).

Ainda para Sabatier (1988, p. 138), 
subsistemas vão além de grupos de interesse, órgãos governamentais e comissões do Legislativo em um único nível de governo, incorporando também

[...] os jornalistas, analistas políticos, cientistas/pesquisadores, personalidades, entre outros que desempenham papéis importantes na geração, disseminação e avaliação de ideias políticas, além de atores em outros níveis de governo que desempenham papéis basilares na formulação e implementação de políticas públicas.

Na maioria dos subsistemas, haverá uma coalizão dominante que é mais bem sucedida em traduzir suas metas em ações governamentais e duas ou três outras subordinadas a ela (WEIBLE, 2005).

Em síntese, Sabatier e Jenkins-Smith (1988, p. 118-20) apresentam pelo menos cinco pressupostos básicos do MCD:

(i) a compreensão dos processos de mudança política e o papel da aprendizagem política a ela associada requer uma perspectiva ampliada de tempo (uma década ou mais); (ii) a unidade de análise mais útil para o estudo dessa mudança é por meio de subsistemas políticos; (iii) esses subsistemas têm que incluir a dimensão intergovernamental; (iv) políticas públicas - ou programas - podem ser conceituadas da mesma maneira por sistemas de crenças (conjuntos de prioridades e assunções causais sobre como realizá-las); e (v) o papel central das informações técnicas e cientificas no processo de mudança política, pois facilita o aprendizado político.

Na sequência, apresentam-se os principais elementos que conformam esse enquadramento teórico.

\section{DESCRIÇÃO DO MODELO}

Recentemente, é preciso registrar, foram elaborados aperfeiçoamentos no modelo, mediante inserção de ajustes em certas categorias de análise, os quais serão aprofundados mais adiante neste tópico.

Figura 1 - Diagrama do MCD (2009)

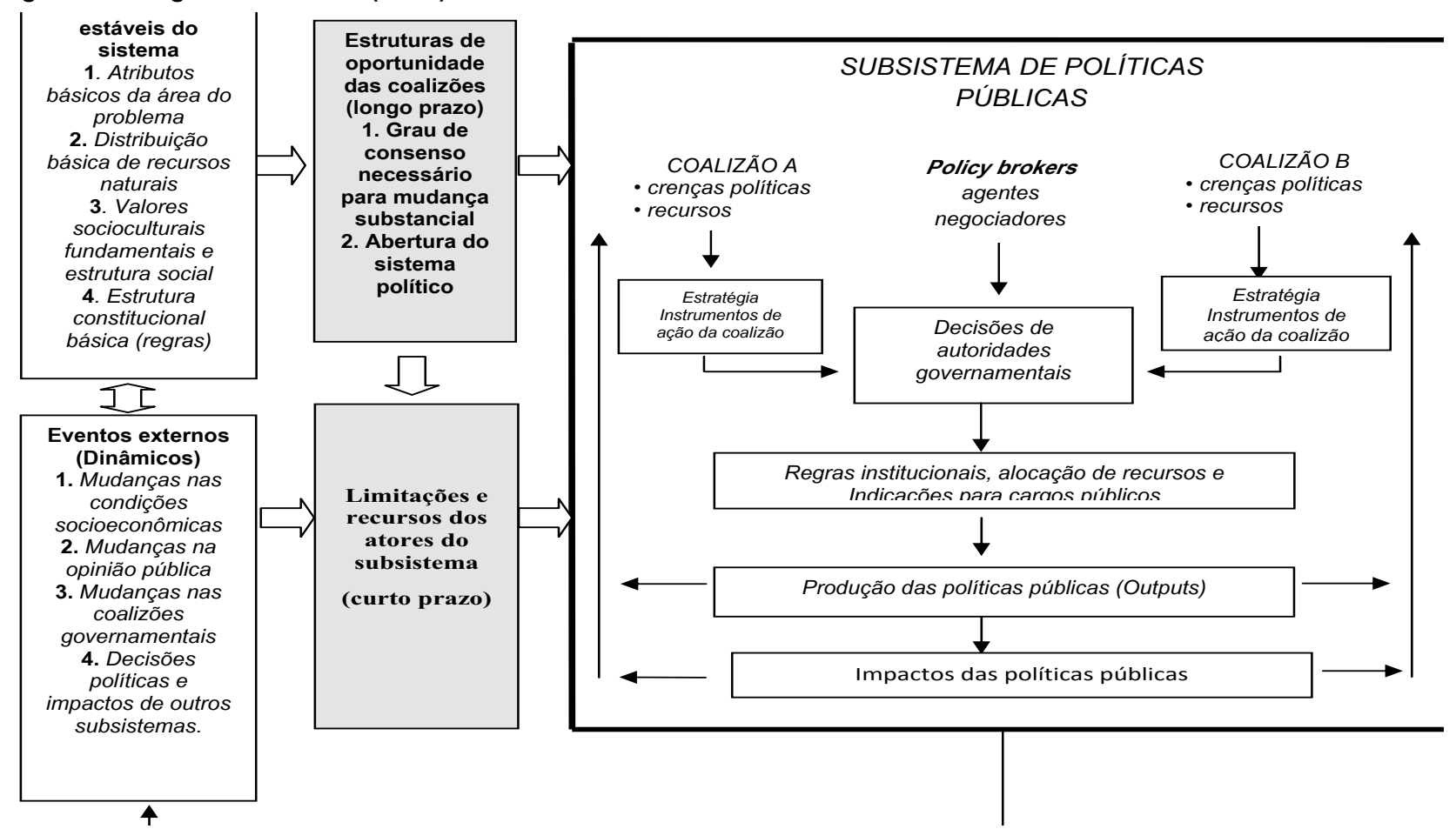

Fonte: Adaptado de: WEIBLE, C. M., SABATIER, P. A.; MCQUEEN, K. Themes and variations: taking stock of the Advocacy Coalition Framework. The Policy Studies Journal, [S. I.], v. 37, n. 1, p. 121-140, 2009. 
A Figura 1 apresenta o quadro analítico do MCD. No lado esquerdo, estão dois conjuntos de elementos exógenos. Um bastante estável (Parâmetros relativamente estáveis do sistema) e outro mais dinâmico (Eventos externos) e que afetam as restrições e oportunidades dos atores dentro do subsistema, geralmente considerados como sendo fatores essencialmente não cognitivos. No lado direito, apresenta-se a dinâmica dos componentes internos do subsistema político no qual geralmente ocorre a dinâmica do policy-oriented learning (fatores cognitivos). A mudança na política pública é interpretada como uma função tanto da competição no interior do subsistema quanto da influência dos eventos exógenos.

\subsection{Eventos exógenos ao subsistema que afetam as mudanças}

\subsubsection{Os parâmetros relativamente estáveis}

Externamente ao subsistema, colocamse os parâmetros relativamente estáveis do sistema como:

a) Os atributos básicos da área do problema ou bem, objeto de uma determinada área de política pública (característicaschave desse problema ou bem e necessidades de intervenção estatal sobre o mesmo);

b) Distribuição básica de recursos naturais: a abundância ou carência de recursos naturais disponíveis em uma sociedade condicionam suas possibilidades de desenvolver diferentes setores econômicos e determinam a viabilidade das opções de políticas públicas.

c) Valores socioculturais fundamentais e estrutura social: significativas mudanças no poder, na influência e nos recursos financeiros de vários grupos sociais normalmente requerem diversas décadas. Para muitos grupos de interesses, os recursos políticos, ou mesmo a falta deles, mudam muito lentamente no tempo e os atores dentro de um subsistema devem levar isso em conta na formulação de suas estratégias de curto e de médio prazo;

d) Estrutura básica das regras constitucionais do sistema político, extremamente difíceis de serem alteradas.

\subsubsection{Os eventos externos (Dinâmicos)}

Os eventos externos ao subsistema são variáveis consideradas mais dinâmicas do que os parâmetros estáveis, pois podem apresentar mudanças no decorrer de uma década ou mais. Os eventos externos ou choques incluem amplas mudanças nas condições socioeconômicas e na opinião pública que podem afetar substancialmente um subsistema a ponto de enfraquecer os pressupostos causais que justificam a atual política pública ou podem alterar o suporte político à determinada coalizão. São fatores mais permeáveis a mudanças, especialmente no espaço de uma década ou mais.

Abrangem também os eventos externos (b) as mudanças nas coalizões governamentais, pois alterações nos padrões políticos partidários são fundamentais no desenho das coalizões, (c) as decisões políticas e impactos de outros subsistemas, e essa variável é importante porque os subsistemas são parcialmente autônomos. As decisões e impactos de outros subsistemas são elementos dinâmicos que afetam um subsistema específico.

A influência desses fatores exógenos no subsistema é mediada pelo grau de consenso necessário para a mudança na política, pelo grau de abertura do regime político, bem como pelas limitações e recursos dos atores do subsistema.

De uma forma geral, os eventos políticos ou econômicos exógenos ao subsistema são essenciais para que agências governamentais alterem sua posição em direção às coalizões concorrentes. Isto 
é, os subsistemas não são imutáveis ante os eventos externos. A Figura 1 mostra que os subsistemas operam dentro de um ambiente político mais amplo e são definidos por parâmetros relativamente estáveis e eventos externos. Além disso, são constrangidos pelas estruturas de oportunidade de longo prazo das coalizões, pelas restrições de curto prazo, pelos recursos disponíveis aos atores do subsistema e pelos eventos de outro subsistema político (FENGER; KLOK, 2001).

\subsection{Eventos Internos ao subsistema}

É no âmbito interno do subsistema que são gerados os programas ou políticas públicas (policy outputs) que frequentemente incorporam elementos ou princípios defendidos por diferentes coalizões. Esses policy outputs são resultados dos processos de interação das coalizões no âmbito do subsistema (Figura 1), e decorrem de mudanças nas regras institucionais e de alocação de recursos impostas pela coalizão vencedora. Nesse sentido, cada coalizão detém determinado conjunto de recursos e opta por estratégias específicas, influenciadas pelo sistema de crenças, para atingir seus objetivos políticos.

Sabatier e Jenkins-Smith (1999) apontam uma série de instrumentos, em tese, disponíveis para atuação das coalizões. Uma coalizão pode tentar influenciar direta e indiretamente as decisões das agências administrativas governamentais, apelando para os tomadores de decisões nos planos legislativo, executivo, judiciário ou intergovernamental.

São exemplos de atuação direta sobre decisões relativas à regulação e orçamento e outras políticas específicas: persuadir agentes públicos por meio de testemunho; a troca de pessoas, dentro de agências e instituições governamentais, o que afeta os recursos políticos de uma coalizão e sua capacidade de influenciar decisões coletivas relativas aos parâmetros da política pública; tornar públicos pela mídia os gaps de desempenho da agência governamental; providenciar relatórios de pesquisa; oferecer estímulos, como propinas ou ofertas de futuros empregos.
São exemplos de atuação indireta por meio dos dirigentes políticos: conduzir revisão sistemática das regras da agência; alterar indicações políticas; caminhar para o litígio; tentar mudanças na legislação; tentar mudanças no orçamento da agência; mudar as preferências políticas pela via eleitoral; e influenciar a opinião pública e, por decorrência, os tomadores de decisão (SABATIER; JENKINS-SMITH, 1993).

Geralmente, a implementação desses instrumentos de ação, visando à alteração de programas e/ou políticas públicas, provoca conflitos pela incompatibilidade entre as ideias e as crenças das coalizões concorrentes. Em regra, a resolução desses conflitos não é tarefa simples, pois esses atores percebem o mundo por meio de lentes distintas e também frequentemente interpretarão as peças das evidências de forma diferente (SABATIER; JENKINS-SMITH, 1988).

Paraisso, existeinternamenteaosubsistema a categoria dos policy brokers (mediadores), constituída por burocratas, parlamentares, juízes de cortes, promotores de Justiça e, mesmo, o conjunto de cidadãos no exercício do voto, que podem atuar no sentido de se chegar a acordos razoáveis que reduzam a intensidade de conflito entre as coalizões no âmbito do subsistema, os quais geralmente não são membros das coalizões. São atores com algum poder para fazer com que compromissos entre coalizões competidoras sejam assumidos e ocorram de fato (FARQUHARSON, 2003). No caso brasileiro, podem ser claramente visualizados como um papel assumido pelo Ministério Público (GURGEL, 2007). Em relação a este ponto, Linquist (2001) chama a atenção para o fato de que instituições de pesquisa também podem atuar como forças de moderação de conflitos.

\section{OS SISTEMAS DE CRENÇAS}

Para Sabatier e Jenkins-Smith (1993), as políticas públicas são conceituadas como sistemas de crenças (beliefs systems). Políticas públicas incorporam teorias sobre como atingir determinados 
objetivos, portanto, envolvem valores, problemas que devem receber as mais altas prioridades, relações causais e percepções da magnitude dos problemas e da eficácia dos instrumentos de intervenção. A possibilidade de relacionar crenças e políticas públicas oferece melhor condição de análise da influência de vários atores no tempo.

Assim, os sistemas de crenças têm a função dupla de moldar uma perspectiva normativa para fundamentar a interpretação e o discernimento de soluções (diagnóstico) em relação a certos fenômenos percebidos. Estes sistemas de crenças são, assim, importantes porque (i) em geral as ações humanas são orientadas pelo sentido e porque (ii), na prática política, a persuasão é um fator central para justificar 0 acesso ao poder e legitimar aqueles que estão no poder (SABATIER, 2007; WEIBLE, 2005).

Neste sentido, propôs-se, um sistema de crenças organizado conforme uma estrutura hierárquica tripartite, disposta segundo 0 grau decrescente de resistência às mudanças (SABATIER; JENKINS-SMITH, 1993; 1988; ARAÚJO, 2007):

- Núcleo duro (deep core) - axiomas normativos e ontológicos fundamentais;

- Núcleo político (policy core) - posições mais importantes sobre a política pública, concernentes às estratégias básicas, às opções programáticas e à teoria de ação para se atingir os valores do deep core no âmbito do subsistema;

- Aspectos instrumentais (secondary aspects) - decisões instrumentais, as medidas e as informações necessárias para implementar o policy core.

\subsection{Núcleo duro (deep core)}

São exemplos de crenças integrantes do núcleo duro (deep core): as concepções sobre a natureza humana, as prioridades relativas a valores fundamentais como direito à vida, dignidade da pessoa humana, liberdade, segurança, poder, conhecimento, saúde, amor, beleza, e outras, os critérios básicos de justiça distributiva, a identidade sociocultural etc. (SABATIER; JENKINS-SMITH, 1993). Em geral, as crenças do núcleo duro são muito resistentes às mudanças basicamente semelhantes a uma conversão religiosa (JENKINS-SMITH; SABATIER, 1994).

\subsection{Núcleo político (policy core)}

Colocam-se no núcleo político (policy core) preceitos normativos essenciais, como a orientação sobre prioridades valorativas básicas e a identificação de grupos sociais ou outras entidades cujo bem-estar é objeto de maior consideração, bem como preceitos com componente empírico substancial, como os referentes a: causas básicas do problema, distribuição adequada de autoridade entre o governo e o mercado e entre os diferentes níveis de governo, prioridades entre os diferentes instrumentos de política pública, preferências políticas de destaque (policy core, policy preferences) etc.

Sabatier e Jenkins-Smith (1988) explicam que as preferências políticas que se inserem no núcleo político (policy core) são aquelas que abrangem todo o subsistema, são altamente salientes e são objeto de conflito relevante por algum tempo. Essas preferências de destaque são crenças que projetam como o subsistema de políticas públicas deveria ser e oferecem um guia para o comportamento estratégico das coalizões, unem aliados e proponentes e dividem oponentes (SABATIER; WEIBLE, 2007).

Deve ser compreendido que as crenças do núcleo político (policy core) servem como primeiro filtro de percepção para os atores de um subsistema de políticas públicas determinarem aliados e oponentes e fontes potenciais de coordenação, informação e conselho (WEIBLE; SABATIER, 2005).

Mudanças nesse nível são difíceis, uma vez que as crenças que integram o núcleo político (policy core) constituem o principal elo interno de ligação das coalizões. Por isso as mudanças no policy core de uma coalizão ou de um programa governamental são geralmente resultantes de fatores 
não-cognitivos (exógenos ao subsistema) (JENKINSSMITH; SABATIER, 1994; SABATIER, 1978).

\subsection{Aspectos instrumentais (secondary aspects)}

A maior parte das preferências concretas em termos da política pública está inserida no nível dos aspectos instrumentais Assim, são exemplos de crenças do nível dos aspectos instrumentais: preferências políticas de menor destaque; crenças de menor amplitude referentes à seriedade de aspectos específicos do problema em locais específicos ou à importância relativa de fatores causais em diferentes momentos e locais; decisões sobre alocação de recursos orçamentários; interpretação de normas; informações sobre o desempenho de programas ou instituições específicas etc. Mudanças nesse nível são relativamente fáceis. É no nível dos aspectos instrumentais que está a maior parte do policymaking administrativo e mesmo legislativo (BONAFONT, 2004).

As mudanças nos aspectos instrumentais ocorrem mediante o chamado policy-oriented learning ou aprendizado orientado à política pública (fatores cognitivos) dentro e entre coalizões de defesa. Essa variável é entendida como vetor fundamental para a análise de mudanças nas políticas públicas (WEYENT, 1988; SABATIER; WEIBLE, 2007)

Para Sabatier (1999), um dos principais elementos de força do MCD está no fato de ele permitir uma visualização relativamente clara quanto ao tipo de mudança que ocorre, se é uma mudança política maior ou menor, ou seja, se houve mudança nos aspectos do núcleo político (policy core) do programa governamental (maior) ou nos aspectos instrumentais (secondary aspects) (menor).

\section{AS HIPÓTESES DO MCD}

Nas primeiras formulações do MCD, foram apresentadas nove hipóteses, e na última versão do trabalho, já havia 16 hipóteses relativas à estrutura das coalizões e de seus sistemas de crenças, às mudanças políticas e ao aprendizado político entre as coalizões (SABATIER; WEIBLE; MCQUEEN, 2009).

\subsection{Hipótese de coalizão (relativas às coalizões de defesa)}

As hipóteses de coalizão são baseadas na premissa de que seu principal elemento aglutinador é a concordância sobre crenças do núcleo político. Essas hipóteses postulam que os grupos de aliados e os de oponentes num dado sistema permanecem estáveis durante períodos de até uma década ou mais. O MCD pressupõe que está essencialmente no núcleo político (policy core) o consenso necessário para a existência da coalizão. Discorda que crenças específicas sejam, na prática, mais relevantes do que crenças abstratas. Os aspectos instrumentais são entendidos como componentes mais negociáveis no âmbito do subsistema.

\subsection{Hipóteses de mudança (relativas às mudanças na política pública)}

As hipóteses de mudança política postulam que uma política pública tende a permanecer por tanto tempo quanto permanecer no poder a coalizão que a criou, tida como coalizão dominante. Segundo essas hipóteses, a única maneira de mudar os atributos do núcleo político de uma política governamental numa dada jurisdição é por meio de algum evento originado fora do subsistema, capaz de alterar substancialmente a distribuição de recursos políticos ou os pontos de vista das coalizões no âmbito desse subsistema. Mas, após alguns ajustes de redação, deixou-se claro que perturbações externas constituem causa necessária, porém não suficiente, para alterações nos atributos fundamentais de um programa governamental e que as autoridades governamentais, em certos casos, têm poder para revisar os programas, mesmo sem mudanças na situação de dominância entre coalizões. 


\subsection{Hipóteses de aprendizado (relativas ao processo de aprendizado orientado às políticas públicas)}

As hipóteses restantes aplicam-se às condições conducentes à aprendizagem política entre sistemas de crenças, ou seja, entre coalizões. Baseando-se na premissa de que as coalizões resistem às mudanças em seu núcleo político ou, mesmo, em aspectos instrumentais importantes de seus sistemas de crenças, esse grupo de hipóteses defende que apenas evidências empíricas bastante sólidas podem conduzi-las a rever pontos de vista (ARAÚJO, 2007; SABATIER, 1987).

Segundo hipóteses da aprendizagem política, apenas evidências empíricas podem levar a mudanças, e é mais provável que essas evidências sejam desenvolvidas e aceitas: (i) em áreas do conhecimento nas quais haja disponibilidade de dados quantitativos e consenso quanto às teorias; (ii) mais no domínio das ciências naturais que nas ciências sociais; (iii) em subsistemas nos quais exista um fórum profissional prestigiado e respeitado; e (iv) em situações envolvendo um nível intermediário de conflito, ou seja, complexo o bastante para justificar o empenho de recursos analíticos, mas não envolvendo conflito normativo direto (FRANÇA, 2002).

\section{AS APLICAÇÕES DO MCD}

\subsection{No exterior}

Os estudos de casos confirmam a utilidade do foco em coalizões de defesa. O MCD não implica na anulação ou demérito das outras teorias, mas sim em um mútuo complemento.

Sabatier e Weible (2007) e Weible, Sabatier e McQueen (2009) mostraram a frequência com que o MCD vem sendo utilizado, dividindo-a nas seguintes categorias: área geográfica, temática, e metodologias utilizadas.

Do ponto de vista geográfico, foram citadas oito áreas: África (Moçambique), Ásia (Taiwan, Coréia do Norte), Austrália, Canadá, América do Sul (Chile,
Brasil), Internacional, Europa, e Estados Unidos. A maioria das aplicações do MCD está concentrada nos Estados Unidos e na Europa, mas com um gradual aumento em outros países.

Em relação às áreas temáticas ou políticas estudadas, foram citados o combate à violência doméstica, políticas de controle do fumo e tabaco, saúde pública, esportes, política sobre resíduos nucleares; política cultural, prevenção de desastres, recursos hídricos, política educacional, transportes, uso e ocupação do solo entre outros assuntos. A maioria das aplicações incidiu nas áreas de política ambiental e energética (WEIBLE; SABATIER; MCQUEEN, 2009; SABATIER; WEIBLE, 2007).

Do ponto de vista metodológico, muitos pesquisadores usaram para fins de coleta, entrevistas, análise do conteúdo de documentos, observação participante, e questionário estruturado, mas em quase a metade de todos os trabalhos analisados os autores não especificaram de forma clara 0 método utilizado. Alguns pesquisadores também combinaram esses métodos, a exemplo de entrevistas e análise documental; questionário e entrevistas; e questionário e análise de conteúdo (WEIBLE; SABATIER; MCQUEEN, 2009).

\subsection{No Brasil}

No caso brasileiro, os primeiros trabalhos com MCD surgiram em 2001, quando Carvalho (2001) examina a evolução da formação da política ambiental no processo de desenvolvimento da indústria metalúrgica no Grande Carajás, na Amazônia Oriental. A pesquisa mostra que, apesar dos esforços da coalizão minoritária de politizar a questão e introduzir algumas reformas políticas pró-ambiente, atores principais da coalizão prómetalurgia (desenvolvimentista) têm sido capazes de dominar o processo de formação política e barrar reformas ambientais significativas. Os resultados têm sido prejudiciais para a conservação ambiental na região (CARVALHO, 2001).

$\mathrm{Na}$ sequência, surgiram trabalhos que versaram sobre a Política de combate às drogas 
ilícitas, França (2002), Segurança Pública e Controle de Armas, Bueno (2005), Biodiversidade e Florestas, Araújo (2007), Turismo, Cavalcanti (2006), Saúde, Gurgel (2007), Educação, Simielli (2008), Esportes, Bueno (2008), Disputas Embates sobre o Projeto de Integração do São Francisco ou da Transposição (Agenda azul) Viana (2011); Regulação do Uso e Ocupação do Solo Urbano Vicente (2012), Política Ambiental (Comparações entre as Agendas verde e marrom) Araujo (2013) entre outros estudos que estão sendo construídos nos diversos núcleos de pesquisas em políticas públicas pelo Brasil afora, demonstrando a espiral crescente da utilização do MCD nos estudos das diversas áreas de políticas públicas no Brasil, corroborando dessa forma a sua consistência interna e externa.

O grosso dos estudos brasileiros que utilizaram 0 MCD sustentou-se nos seguintes pressupostos metodológicos: a maioria empregou a análise documental (audiências públicas) e entrevistas para a coleta de dados, e, para organizar e compreender os dados coletados, a técnica da análise de conteúdo prevaleceu sobre as outras técnicas de análise de dados.

Demonstra-se, assim, o predomínio da pesquisa qualitativa e do estudo de caso sobre a pesquisa quantitativa na decisão dos pesquisadores na hora de escolher a estratégia metodológica a ser seguida.

Estudos sobre mudanças nos arranjos institucionais de regulação que conformam a política de uso e ocupação do solo urbano estão sendo desenvolvidos. Está-se buscando aplicar o MCD, para explicar o processo pelo qual as mudanças ocorreram neste subsistema político, analisando como as coalizões se estruturaram e quais predominaram nas tomadas de decisões ao longo do tempo. Portanto, os processos decisórios devem ser estudados para compreender como os atores em diferentes níveis governamentais tomam decisões, como agentes públicos e privados interagem na tomada de decisões e quais os fatores e recursos influenciam as suas decisões e o impacto dessas decisões sobre os instrumentos de regulação da política fundiária do Distrito Federal (VICENTE, 2010; 2012).

Essa abordagem é interessante porque mudanças nos instrumentos que regulam o uso e ocupação do solo urbano são resultados de escolhas feitas por indivíduos, empresas, organizações não-governamentais e governos (THIHA; HONDA, 2007). No tocante a isto, será necessário examinar as crenças políticas das elites das coalizões organizacionais e suas estratégias para influenciar decisões, mudanças e a implementação da política em tela.

Apesar da espiral crescente da sua aplicação em pesquisas no contexto brasileiro, o aproveitamento do MCD ainda é tímido no país se comparado com os de países da Europa e da América do Norte, o que torna mais interessante o seu estudo.

\section{CONCLUSÃO}

\subsection{Virtudes}

Desde 1988 o MCD tem se desenvolvido como um dos mais promissores modelos de estudos de políticas públicas (PRINCEN, 2007; SHU-HSIANG, 2005; CHEN, 2003; ELLISON, 1998; SCHALAGER, 1995; JENKINS-SMITH; SABATIER, 1994; SABATIER, 1987; 1988). Tivemos centenas de publicações produzidas pelos pesquisadores mundo afora, e abrangendo diversas temáticas como política nuclear, políticas de esportes, políticas ambientais, combate à violência doméstica, políticas antidrogas, saúde, políticas de uso e ocupação de terras, entre outras questões (SABATIER; WEIBLE, 2007).

A estrutura do MCD está focada em fatores que podem explicar como as mudanças políticas ocorrem dentro de um subsistema de política específica. Tais fatores incluem a força e os eventos externos ao subsistema, como condições socioeconômicas gerais e os resultados eleitorais. Mas o mais importante é que o quadro analítico oferece a possibilidade de explicar a evolução da política como o resultado de aprendizagem política, 
isto é, analisando a importância da busca contínua pelos atores de coalizões em competição de novas evidências, novos argumentos e novas táticas e estratégias que possam traduzir suas crenças em programas de ação propostos para a política governamental (SABATIER; MCLAUGHLIN, 1988).

Dessa forma, Sabatier e Weible (2007) entendem que o policymaking e o decisionmaking são resultados de uma ampla negociação entre coalizões, que incluem atores de dentro e de fora da estrutura do governo, e que atuam dentro de um determinado subsistema de políticas públicas.

0 processo político, então, deve ser entendido no contexto de redes e comunidades políticas, pois da análise do conjunto de convicções, valores, ideias e fatores socioeconômicos elucidamse quais critérios foram utilizados na construção do processo de definição de políticas.

Outro fator importante é que o MCD assume explicitamente que a maioria das coalizões inclui atores dos vários níveis de governo, superando a visão reducionista dos triângulos de ferro, caracterizada por uma tendência a limitar a participação nas decisões políticas às agências administrativas, comissões do Legislativo e grupos de interesse em um único nível de governo, além do descuido com os atores não-estatais. Primeiro, porque praticamente todos os programas nacionais dependem fortemente dos governos subnacionais para a sua implementação efetiva. Segundo, porque as transferências intergovernamentais constituem porcentagem significativa da maioria dos orçamentos dos governos subnacionais.

$E$, em terceiro, porque as organizações governamentais e não-governamentais dos estados e municípios formam uma porcentagem substancial de grupos que influenciam as decisões políticas (SABATIER; JENKINS-SMITH, 1994).

Portanto, o desenvolvimento de um quadro analítico para compreender mudanças nas políticas públicas é uma oportunidade para examinar em detalhe uma série de casos em diferentes contextos políticos e nas mais variadas temáticas.
Todavia, o bom emprego do MCD nessa variedade de políticas - sabendo quando é apropriado ou não para análise de uma temática e quais são seus limites - requer considerável julgamento por parte do pesquisador ou do analista político. Analistas políticos e pesquisadores que irão utilizar o MCD devem ser capazes de adaptar as suas análises e seus estilos e pesquisas ao contexto político e, assim, contribuir mais eficazmente para a injeção de conhecimento compartilhado no processo decisório (HEINTZ JR.; JENKINS-SMITH, 1988).

Em suma, o MCD assume que as coalizões são atores fundamentais na análise das ações governamentais por três razões: em primeiro lugar, porque elas constituem um arco de alianças que é capaz de sustentar politicamente os programas e as políticas públicas tanto no nível partidário e parlamentar quanto na sociedade e entre os atores burocráticos; em segundo lugar, porque as coalizões têm o conceito de mobilização como uma característica intrínseca a elas, dado que buscam constantemente apoiadores para a sua causa, a fim de aumentar a sua solidez; e, por fim, as coalizões são importantes por estabelecerem relevantes espaços de debate e aprendizado em relação às políticas públicas, possibilitando antecipar mudanças e corrigir erros (SABATIER, 2007).

Schlager (1995) aponta que os modelos que estudam as comunidades políticas (policy communities), redes políticas (policy networks) e coalizões de defesa (advocacy coalition) são os que melhor descrevem e explicam o complexo e dinâmico processo político na sociedade moderna, e, por sua vez, são os mais utilizados nos Estados Unidos e em países Europeus.

\subsection{Limitações}

O MCD tem sido criticado por ter um viés concernente a sistemas políticos pluralísticos como 0 dos Estados Unidos. Esta critica está sendo vencida, o que mostra que o ACF pode ser utilizado em quase todos os formatos políticos e culturas, incluindo 
regimes autoritários em países em desenvolvimento. Com relação à crítica de que o modelo tem tido uma forte associação com políticas ambientais e de recursos naturais, o levantamento feito por Sabatier e outros pesquisadores demonstrou que o MCD pode ser aplicado em quase todos os domínios de políticas públicas (WEIBLE; SABATIER; MCQUEEN, 2009).

Críticos da Modelo de Coalizões de Defesa também argumentam que as crenças apenas são ecos das estruturas econômicas que, em última instância, definem os interesses.

Porém, para Sabatier, é quase impossível identificar os verdadeiros interesses dos agentes. Sabatier considera mais fácil identificar as convicções deles, porque eles precisam se posicionar nos debates políticos (BUENO, 2005).

John (1998) critica o modelo, afirmando que, em troca de sua clareza e simplicidade, a MC peca por não explicar vários aspectos importantes de mudanças na política, a saber: como as coalizões realmente traduzem suas crenças em ação governamental, e como agências governamentais são mobilizadas como parte das coalizões. Nem 0 modelo fornece um enquadramento útil para examinar como os brokers realizam suas empreitadas.

Segundo John (2003), fica a sugestão de aprofundamento nesses pontos, o que esclareceria ainda mais 0 porquê e como certas mudanças nas políticas públicas ocorrem em determinados períodos de tempo, bem como quem poderia fazer o papel de catalisador dessas políticas e com que tipo de recursos e táticas. Para esse autor, poucos aprofundamentos nos aspectos supracitados podem tornar o MCD limitado em ideias e não tão integrador quanto postulam os seus idealizadores.

Os autores do MCD rebatem as críticas de John afirmando que a literatura do MCD não é limitada em ideias, relevância ou esforço intelectual. $\mathrm{Na}$ verdade, estudos mostram que a literatura do MCD continua se expandindo em aplicações em diversas áreas geográficas e em temáticas das mais variadas, fornecendo uma lente útil, especialmente em conjunto com outras teorias e modelos para explicar o processo político e suas mudanças (WEIBLE; SABATIER; MCQUEEN, 2009, p. 136).

É oportuno, portanto, concluir a presente explanação sobre o Modelo de Coalizões de Defesa com a ressalva de que, em todos os estudos de casos em que foi aplicado, várias revisões foram necessárias. Assim, 0 aspecto fundamental a ser ressaltado em sua utilização não é provar sua infalibilidade empírica, mas que este quadro analítico representa uma das formas de explicar os padrões de mudança em um mundo em que as políticas públicas são cada vez mais intersetoriais e transversais (SERRA, 2004; MENICUCCI, 2002).

Recomenda-se a todos os pesquisadores interessados no MCD a explorar as ramificações políticas e comportamentais deste modelo, testar, aplicar e expandir as suas hipóteses (SABATIER; WEIBLE, 2007).

\section{REFERÊNCIAS}

ARAÚJO, S. M. V. G. Coalizões de advocacia na formulação da política nacional de biodiversidade e florestas. 2007. 332 f. Dissertação (Mestrado em Ciência Política) - Instituto de Ciência Política, Universidade de Brasília, Brasília, DF, 2007.

Política ambiental no Brasil no período 1992-2012: um estudo comparado das agendas verde e marrom. 2013. 458 f. Tese (Doutorado em Ciência Política) - Instituto de Ciência Política, Programa de Pós-Graduação em Ciência Política, Universidade de Brasília, Brasília, DF, 2013.

BONAFONT, L. C. Redes de políticas públicas. Madri: Centro de investigaciones Sociológicas, 2004.

BUENO, L. A Aplicação da. Advocacy Coalition Framework (ACF) na análise da evolução da política pública de controle de armas no Brasil. In: ENCONTRO ANUAL DA ANPOCS, 29., 2005, Caxambu, MG. Anais... Caxambu, MG, 2005. 
Políticas publicas do esporte no Brasil: razões para 0 predomínio do alto rendimento. 2008. 200 f. Tese (Doutorado Administração Pública e Governo) - Escola de Administração de Empresas de São Paulo, Fundação Getúlio Vargas, 2008.

CAVALCANTI, P. A. B. Um olhar crítico sobre o Conselho Nacional de Turismo: articulação do setor, legitimidade e auto-interesse na construção das Políticas Públicas. 2006. Dissertação (Mestrado) - Escola de Administração de Empresas, Fundação Getúlio Vargas, São Paulo, 2006

CARVALHO, G. O. Metallurgical developmen $t$ in the Carajas area: A case study of the evolution of environmental policy formation in Brazil. Society and Natural Resources, [S. I.], v. 14, p. 127-43, 2001.

CHEN, P. Advocating online censorship. Australian Journal of Public Administration. Sydney, v. 62, n. 2, p. 41-64, jun. 2003.

ELLISON, B. Intergovernmental relations and the advocacy coalition framework: the operation of federalism in Denver water politics. Publius: the Journal of Federalism, Oxford, v. 28, n. 4, p.35-54, 1998.

ENGEL, F. Analyzing policy learning in European Union policy formulation: the advocacy coalition framework meets new-institutional theory. Belgium: European Political and Administrative Studies, 2007. (Bruges Political Research Papers, n. 5).

FARQUHARSON, K. Influencing policy transnationally: Pro-and Anti-Tobacco Global Advocacy Networks. Australian Journal of Public Administration, [S. I.], v. 62, n. 4, p. 80-92, dec. 2003.

FENGER, M.; KLOK P-J. Interdependency, Beliefs, and Coalition Behavior: A contribution to the Advocacy Coalition Framework. Policy Sciences, [S. I.], v. 34, p.157-170, 2001.

FRANÇA, E. F. Coalizões de advocacia na formulação da política de controle de drogas no Brasil, no período 1998/2000. 2002 Dissertação (Mestrado) - Universidade de Brasília, Brasília, DF, 2002.
GURGEL, G.D. I. A pesquisa científica na condução de políticas de controle de doenças transmitidas por vetores. 2007. 310 f. Tese (Doutorado em Saúde Pública) - Centro de Pesquias Aggeu Magalhães, Fundação Oswaldo Cruz, Recife, 2007.

HAAS, E. B. When knowledge is power: three models of change in international organizations. Ewing: University of California Press, 1991.

HEINTZ JR. H. T.; JENKINS-SMITH, H. C. Advocacy coalitions and the practice of policy analysis. Policy Sciences, [S. I.], v. 21, p.263-277, 1988.

HOWLETT, M.; RAMESH, M. Policy subsystem configura tions and policy change: Operationalizing the postpositivist analysis of the politics of the policy process. Policy Studies Journal, [S. I.], v. 26, n. 3, p. 466-481, 1998.

JENKINS-SMITH, H. C.; SABATIER. P. A. Evaluating the advocacy coalition framework. Jounal of Public Policy. Cambridge, v. 14, n. 2, p. 175-203, 1994.

JOHN, P. Is there life after policy streams, advocacy coalitions, and punctuations: using evolutionary theory to explain policy change? The Policy Studies Journal, Malden, v. 31, n. 4, p. 481-498, 2003.

Analysing public policy. Londres: Pinter, 1998. (Critical Political Studies).

LINQUIST, E. A. Discerning policy influence: framework for a strategic evaluation of IDRCsupported research. [S. I.: s. n.], 2001, Disponível em:<http://www.idrc.ca/uploads/user-S//1095694789 10359907080discerning/_policy.pdf>. Acesso em: 9 jan. 2009.

MENICUCCI, T. M. G. Intersetorialidade, o desafio atual para as políticas sociais. Pensar BH/Política Social, Belo Horizonte, ed. Temática, n. 3, p. 10-13, maio/jul. 2002.

PRINCEN, S. Advocacy coalitions and the internationalization of public health policies. Journal of Public Policy, [S. I.], v. 27, n. 1, p. 13-33, 2007.

SABATIER, P. A. (Ed.). Theories of the Policy Process. 2. ed. Boulder: Westview Press, 2007. 
An advocacy coalition framework of policy change and the role of policy-oriented learning therein. Policy Sciences, [S. I.], v. 21, p.129-168, 1988.

Knowledge, policy-oriented learning, and policy change: an advocacy coalition framework. Science Communication, [S. I.], v. 8, n. 4, p. 649692, 1987.

The acquisition and utilization of technical information by administrative agencies. Administrative Science Quarterly, [S. I.], v. 2, n. 3, p. 396-417, sept. 1978.

The advocacy coalition framework: an assessment. In: SABATIER, P. A. (Ed.). Theories of the Policy Process. Boulder: Westview Press, 1999. p. 117-166.

The Advocacy coalition framework: revisions and relevance for Europe. Journal of Europe Public Policy, [S. I.], v. 5, n. 1, p. 98-130, 1998.

SABATIER, P. A.; JENKINS-SMITH, H. (Eds.). Symposium on policy change and policy-oriented learning. Policy Sciences, v. 21, n. 2-3, p.123-177, 1988.

The advocacy coalition framework: an a ssessment, revisions, and implications for scholars anda practioners. In: SABATIER, P. A.; JENKINS-SMITH, H. C. (Eds.). Policy change and learning: an advocay coalition approach. Boulder: Western Press, 1993. p. 211-235.

The advocacy coalition framework: an assessment. In: SABATIER, P.A (Ed.). Theories of the Policy Process. Boulder: Westview Press, 1999. p. 117-166.

; MCLAUGHLIN, S. M. Belief congruence of governmental and interest group elites with their constituencie. American Politics Quarterly, [S. I.], v. 16, n. 1, p. 61-98, jan. 1988.

; WEIBLE, C. M. The advocacy coalition framework: innovations, and clarifications. In: SABATIER, P. A (Ed.). Theories of the Policy Process. 2. ed. Boulder: Westview Press, 2007. p. 189-220.
SCHLAGER, E. Policy making and collective action: Defining coal itions within the advocacy coalition framework. Policy Sciences, [S. I.], v. 28, p.243-270, 1995.

SERRA, A. La gestión transversal: expectativas y resultados. In: CONGRESO INTERNACIONAL DEL CLAD SOBRE LA REFORMA DEL ESTADO Y DE LA ADMINISTRACIÓN PÚBLICA, 9., 2004, Madrid. Anais... Madrid, España, 2004.

SHU-HSIANG, H. Advocacy coalitions and policy change on nuclear power utilization in Taiwan. The Social Science Journal, [S. I.], v. 42, p. 215-229, 2005.

SIMIELLI, L. E. R. Coalizões em educação no Brasil: a pluralização da sociedade civil na luta pela melhoria da educação pública. 2008. Dissertação (Mestrado em Administração) - Escola de Administração de Empresas, Fundação Getúlio Vargas, São Paulo, 2008.

THIHA, W. E. L.; HONDA, K. Biophysical and policy drivers of landscape change in a central Vietnamese district. Environmental Conservation, Switzerland, v. 34 n. 2, p. 164-172, 2007.

VIANA, L. B. F. Entre o abstrato e o concreto: Legados do embate sobre o Projeto de Integração do São Francisco ou da Transposição. 2011. 200 f. Dissertação (Mestrado em Ciência Política) - Instituto de Ciência Política, Universidade de Brasília, Brasília, DF, 2011.

VICENTE, V. Análise de mudanças institucionais na política de ordenamento territorial urbano no Distrito Federal (1991-2009). 2012. 231 f. Tese (Doutorado em Administração) - Programa de Pós Graduação em Administração, Universidade de Brasília, Brasília, DF, 2012.

Mudanças nos arranjos institucionais de regulação do uso do solo conformados nos planos diretores de ordenamento territorial no Distrito Federal - 1988-2008: atores e conflitos. 2010. Qualificação de Projeto de Tese (Doutorado em Administração) - Programa de Pós Graduação em Administração, Universidade de Brasília, Brasília, DF, 2010. 
WEIBLE, C. M. Beliefs and perceived influence in a natural resource conflict: an advocacy coalition approach to policy networks. Political Research Quarterly, v. 58, n. 3, set. 2005.

Coalitions, science, and belief change: Comparing adversarial and collaborative policy subsystems. The Policy Studies Journal, Malden, v. 37, n. 2, p. 195-212, 2009.

Expert-based information and policy subsystems: A review and synthesis. The Policy Studies Journal, Malden, v. 36 , n. 4, p. 615-635, 2008.

; SABATIER, P. A. Comparing policy networks. Marine protected areas in California. The Policy Studies Journal, Malden, v. 33, n. 2, p. 181-201, 2005.

; _ MCQUEEN, K. Themes and variations: taking stock of the Advocacy Coalition Framework. The Policy Studies Journal, Malden, v. 37 , n. 1, p. 121-140, 2009.

WEISS, C. H. Ideology, interests and information: the basis of policy positions. In: CALLAHAN, D.; JENNINGS, B. (Eds.), Ethics, social sciences and policy analysis. New York: Plenum Press, 1983.

. Research for policy's sake: The enlightenment function of social research. Policy Analysis, [S. I.], n. 3, p. 531-545, 1977.

WEYENT, J. Is there policy-oriented learning in the analysis of natural gas policy issues? Policy Sciences, [S. I.], v. 21, p. 239-262, 1988.

\section{Victor Manuel Barbosa Vicente}

Administrador

Doutor em Administração pela Universidade de Brasília (UnB)

Professor da Universidade Federal de Uberlândia (UFU) e Pesquisador junto ao Centro de Estudos Avançados de Governo e Administração Pública (CEAG) da Universidade de Brasília (UnB)

E-mail: victorvicente.unb@gmail.com

Universidade Federal de Uberlândia - UFU

Av. João Naves de Ávila 2121, Campus Santa Mônica, CX 593
- Uberlândia - MG

CEP: 38408-100

Universidade de Brasília - UnB

Campus Universitário Darcy Ribeiro, Brasília - DF CEP: $70910-900$ 